\title{
Research on DC motor speed control system based on PID
}

\author{
Huang Jian \\ XiJing University, Xi'an 710123, China; \\ 565200245@qq.com
}

Keywords: Timer; PWM wave; PID control

\begin{abstract}
In engineering design and experiment, the speed of DC motor is often controlled. The common way is to generate PWM wave through timer, and adjust the speed of motor according to duty cycle. In theory, the velocity is proportional to the duty ratio. But in practice, because of the resistance, friction, motor performance parameters and so on, it often appears in a certain fixed duty ratio, the motor speed is uncertain. At this time, the speed of the DC motor is controlled by PID, and the speed of the DC motor is constant by adjusting the ratio, differential and integral coefficient.
\end{abstract}

\section{Introduction :}

In the engineering practice, the speed of the DC motor is often controlled. The common practice is to generate PWM waves through the timer of the microprocessor, adjust the width of the positive pulse width, and control the speed of the DC motor. The speed is basically proportional to the width of the positive pulse. But if the curve is studied carefully, it is not necessarily in line with the linear relationship. Therefore, in order to get a constant velocity value, the method of automatic control must be introduced. The PID adjustment is suitable for adjusting the speed of the motor. By adjusting the ratio, differential and integral coefficient, the duty ratio of the PWM wave is adjusted, and the speed is constant.

\section{Hardware Design}

The system block diagram is shown in Figure 1. In Figure 1, the main control adopts STM32F103ZET6, generates PWM wave through timer TIMER1, controls TB6612 to drive DC motor, and uses TIMER2 to measure the speed of motor, then feedback the measurement value to the microprocessor, and gets the deviation by comparing the predetermined value and measured value. The output of the PWM wave is controlled by the deviation. Use 1.44 inch TFT to display the relevant information and adjust the speed of the motor with key buttons. 


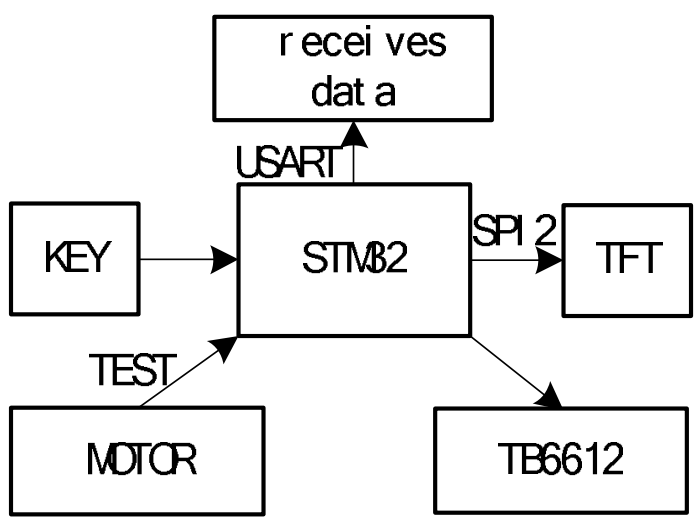

Fig 1 System block diagram

\section{Software Design}

In this design, it is programmed in the $\mathrm{C}$ language under KEIL 5. The measurement of speed is realized by the timer, and the PID adjustment is carried out regularly in the timer.

The incremental PID regulation code is as follows int TIM1_UP_IRQHandler(void)

\{

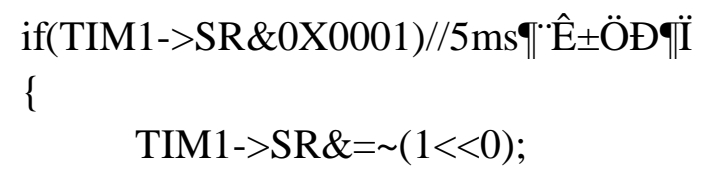

Encoder=Read_Position(2);

Moto=Position_PID(Encoder,Target_Position);

\}

else

if(++Flag_Velocity>2)

Flag_Velocity $=1$;

Encoder=Read_Velocity(2);

if $($ Encoder $<0)$

Encoder $=0$;

Moto=Incremental_PI(Encoder,Target_Velocity); \}

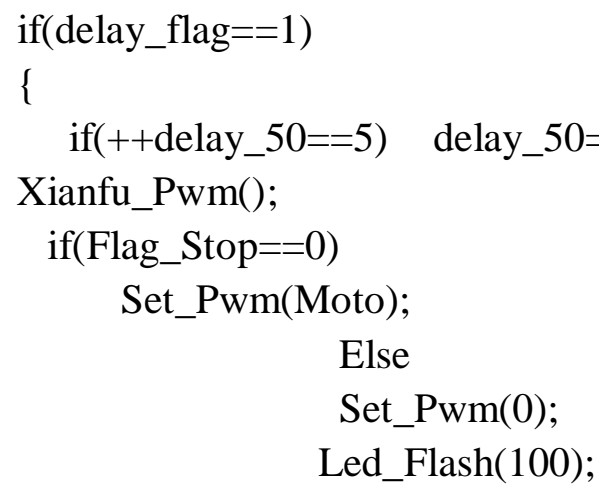

$\operatorname{Key}()$;

Voltage=Get_battery_volt();

\}

return 0 ; 
Key control :

void Key(void)

\{

int tmp,tmp2,Position_Amplitude=260,Velocity_Amplitude=15;

tmp=click_N_Double(50);

if(Menu_MODE==1)

\{

if $(\mathrm{tmp}==1)$ Target_Position+=Position_Amplitude;

if $(\mathrm{tmp}==2)$ Target_Position-=Position_Amplitude;

\}

else

\{

if $(\mathrm{tmp}==1)$ Target_Velocity+=Velocity_Amplitude;

if $(\mathrm{tmp}==2)$ Target_Velocity-=Velocity_Amplitude;

\}

if(Target_Velocity $>50)$ Target_Velocity $=50 ; \quad$ if $($ Target_Velocity $<0)$ Target_Velocity $=0$; tmp2=Long_Press () ;

if $(\operatorname{tmp} 2==1)$ Flash_Send=1;

\}

Pwm :

void Xianfu_Pwm(void)

\{

int Amplitude=7100;

if(Moto<-Amplitude)

Moto=-Amplitude;

if(Moto>Amplitude)

Moto=Amplitude;

\}

\section{Summary}

This paper describes the method and principle of using PID to adjust the speed of DC motor, and gives the block diagram of the system. The working principle of the machine is explained, and a timer is used to measure the speed of the motor in real time. By comparing the deviations, the deviation is adjusted by PID, and the accurate values of P, I and D are obtained through many experiments.

\section{Reference :}

[1] Lu Yi, Qi Zhidong. A speed regulation based on fractional order PID DC motor AGV control system [J]. modern electronic technology, 2017,40 (3): 127-130.

[2] Wu Tongkun, Zhou Chaoying, Xie Peng. Design of a new variable spray system for remote control based on PWM [J]. research on agricultural mechanization, 2017,7:76-82.

[3] Chen Jiang. Research on the simulation of Brushless DC motor system [J]. modern radar, 2011, 
33 (7): 56-59.

[4] Zhao Zhitao, Jia Yanbin, Zhao Zhicheng, he Hou Sheng. Permanent magnet brushless DC motor speed control system of improved IMC- PI control [J]. fire and command and control, 2016,41 (9): 70-79.

[5] Jiabing Liu, Li Zhihong, Ren Yanfeng. Modeling and Simulation of Brushless DC motor fuzzy [J]. control engineering of PI system, 2008,15 (S1): 125-133.

[6] Wang Jun, Feng Nenglian, Zhang Chunqiang, Zhang Xingyu. Brushless DC motor fuzzy and PI double closed loop speed control system research [J]. Journal of Anhui Agriculture University, 2016,43 (4): 662-666.

[7] Wen Jiabin, machen Wei. Fuzzy PI control system for brushless DC motor design [J]. Journal of motor and control, 2016, 20 (3): 102-108. 